\title{
Redução da Densidade Mineral Óssea em Mulheres na Menacme com Prolactinoma
}

\section{artigo original}

\author{
ISABELA BUSSADE \\ ERIKA C.O. NALIATO \\ Laura Maria C. Mendonça \\ ALICE H.D. VIOLANTE \\ MARIA LUCIA F. FARIAS
}

Serviço de Endocrinologia,

Hospital Universitário Clementino Fraga Filho, Faculdade de Medicina da Universidade Federal Rio de Janeiro, RJ.
Recebido em 06/11/06

Revisado em 29/05/07 e 01/08/07

Aceito em 22/10/07
RESUMO

A hiperprolactinemia tumoral e conseqüente hipogonadismo têm sido associados à osteoporose. Avaliamos a densidade mineral óssea (DMO) por absortometria com dupla fonte de RX em 24 mulheres entre 18 e 49 anos, com prolactinoma (15 macro e 9 micro). Utilizamos teste $t$ de Student não pareado ou Mann-Whitney para comparar subgrupos, e teste de Spearman para correlações. O maior acometimento foi de coluna lombar, onde $20,83 \%$ das pacientes tinham Z-escore $\leq-2$ DP. Não detectamos diferenças densitométricas entre macro e microprolactinomas, nem entre pacientes com prolactina normal versus as hiperprolactinêmicas. A DMO e o Z-escore na coluna foram maiores nas pacientes $\mathrm{com} \geq 8$ ciclos menstruais no ano anterior à densitometria versus as oligoamenorréicas $(p=0,030)$. O número de ciclos/ano correlacionou-se com a DMO na coluna $(r=0,515, p=0,017)$, e o índice de massa corporal, com a DMO em colo femural $(r=0,563, p=0,006)$ e fêmur total $(r=0,529, p=0,011)$. Conclusões: Em nossa amostra de mulheres jovens com prolactinoma, 20,83\% têm densidade óssea abaixo do esperado para a idade. O maior acometimento de regiões ricas em osso trabecular, como as vértebras, sugere a participação do hipogonadismo na gênese da doença óssea. Independentemente dos valores séricos de prolactina, o retorno dos ciclos menstruais parece ser o melhor índice de bom controle dessas pacientes. (Arq Bras Endocrinol Metab 2007;51/9:1522-1527)

Descritores: Prolactina; Prolactinoma; Densidade óssea; Osteoporose; Amenorréia

\section{ABSTRACT}

Decreased Bone Mineral Density in Pre-Menopause Women with Prolactinoma.

Tumoral hyperprolactinemia and consequent hypogonadism have been associated with osteoporosis. Bone mineral density (BMD) was measured by dual-energy $\mathrm{RX}$ absorptiometry in 24 patients with prolactinoma (15 macro and 9 micro adenomas; age range $=18$ to 49 years). Student unpaired $t$ or Mann-Whitney tests were used to compare groups, and Spearman test studied correlations. Lumbar spine (LS) was the most affected, as LS Z-score was $\leq-2$ SD in $20.83 \%$ of the patients. No difference was found in densitometric parameters for the comparison between macro and microprolactinoma, or those with normal prolactin versus hyperprolactinemia. LS BMD and LS Z-score were higher in the patients with $\geq 8$ menstrual cycles in the preceding year then in those with oligoamenorrhea $(p=$ $0.030)$. The number of cycles was correlated to LS BMD $(r=0.515, p=0.017)$ and body mass index to femoral neck BMD $(r=0.563, p=0.006)$ and total femur BMD $(r=0.529, p=0.011)$. Conclusions: Decreased bone mineral density was detected in $20.83 \%$ of our young patients with prolactinoma. The great involvement of trabecular bone skeletal regions, such as vertebrae, suggests the participation of hypogonadism in the pathogenesis of bone disease. Irrespective of prolactin levels, return to normal menses seems the best index of good control. (Arq Bras Endocrinol Metab 2007;51/9:1522-1527)

Keywords: Prolactin; Prolactinoma; Bone density; Osteoporosis; Amenorrhea 
A ASSOCIAÇÃO ENTRE DEFICIÊNCIA estrogênica e diminuição da densidade mineral óssea (DMO) já é bem estabelecida em mulheres após a menopausa (1), tendo sido também reconhecida em mulheres jovens com hipoestrogenismo (2). Os efeitos da deficiência estrogênica no esqueleto são caracterizados por aumento da remodelação óssea, sendo a taxa de reabsorção óssea desproporcionalmente maior que a capacidade de formação, o que ocasiona perda óssea progressiva e osteoporose. O osso trabecular (principal componente dos corpos vertebrais) é mais acometido que o osso cortical (predomina na diáfise de ossos longos e colo do fêmur); desta forma, as fraturas de antebraço distal e o colabamento vertebral antecedem as fraturas do fêmur proximal (1).

Há 20 anos reconhece-se a influência negativa da hiperprolactinemia no osso (3-5). A conseqüente diminuição da massa óssea pode acontecer em fases precoces da vida, acometendo mulheres jovens, ou seja, em uma faixa etária onde seria esperado haver incremento ou estabilização da massa óssea. Essa perda óssea precoce, se não diagnosticada e tratada, pode aumentar o risco de fraturas, acrescentando maior morbidade e mortalidade à doença de base.

A hiperprolactinemia causa hipogonadismo pela inibição hipotalâmica da produção de hormônio liberador do hormônio luteinizante (LHRH), com conseqüente decréscimo na secreção de hormônio luteinizante e hormônio folículo-estimulante (6). Esse hipogonadismo funcional, percebido como oligo-amenorréia (atraso menstrual ou interrupção das menstruações), antecipa o hipoestrogenismo para a fase prémenopausa, com todas as conseqüências sobre o osso. O tempo de duração dos distúrbios menstruais parece ser um dos fatores cruciais na perda de massa óssea apresentada por essas pacientes (7).

Por outro lado, tem sido considerada a hipótese de ação direta da prolactina nas células ósseas, independentemente do hipoestrogenismo. Já foi demonstrado que a administração de prolactina em ratos diminui em $30 \%$ os níveis de fosfatase alcalina (secretada por osteoblastos) e leva à diminuição da ossificação endocondral (8). Apesar desta demonstração in vivo, evidências clínicas da ação direta da prolactina no osso ainda são inconsistentes.

Diversos outros fatores podem interferir na homeostase óssea dessas pacientes, como a co-existência de hiperparatireoidismo primário, se o prolactinoma for uma das manifestações da neoplasia endócrina múltipla tipo I (9) ou hiperparatireoidismo secundário à baixa ingestão de cálcio e insuficiência de vitamina D (10). A deficiência de outras trofinas hipofisárias também deve ser considerada, principalmente se esses hormônios não estiverem sendo repostos de maneira fisiológica.

O objetivo deste estudo foi avaliar a massa óssea de mulheres no menacme com diagnóstico confirmado de prolactinoma e relacionar a doença óssea ao controle clínico da hiperprolactinemia.

\section{PACIENTES E MÉTODOS}

Trata-se de um estudo descritivo de corte transversal. O estudo foi aprovado pelo Comitê de Ética em Pesquisas do Hospital Universitário/ Faculdade de Medicina da Universidade Federal do Rio de Janeiro (UFRJ). Avaliamos, entre as pacientes acompanhadas no ambulatório de Pesquisa em Hiperprolactinemia do Serviço de Endocrinologia do Hospital Universitário Clementino Fraga Filho (HUCFF) da UFRJ, aquelas que tivessem entre $18 \mathrm{e}<50$ anos e prolactinoma. Esse diagnóstico foi estabelecido mediante no mínimo duas dosagens de prolactina elevadas, sendo pelo menos uma acima de $100 \mathrm{ng} / \mathrm{ml}$ pelo método de eletroquimioluminescência (valor de referência 6 a $30 \mathrm{ng} / \mathrm{ml}$ ) e tumor hipofisário confirmado por tomografia computadorizada ou ressonância magnética. Todas as pacientes com macroprolactinoma, submetidas ou não à cirurgia transesfenoidal, são regularmente testadas para insuficiência corticotrópica e somatotrópica mediante o teste de hipoglicemia insulínica. Pacientes com hipotireoidismo e/ou deficiência de cortisol poderiam ser incluídas no estudo, desde que estivessem repondo doses fisiológicas de levotiroxina e/ou glicocorticóide. Os critérios de exclusão foram: deficiência de $\mathrm{GH}$, níveis séricos de $\mathrm{FSH}>30$ $\mathrm{mU} / \mathrm{l}$ caracterizando menopausa espontânea, qualquer doença crônica ou uso regular de medicamentos que pudessem interferir nos níveis de prolactina e/ou na homeostase óssea. A rotina de exames incluiu cálcio e PTH, avaliação da função renal (creatinina sérica) e hepática (albumina, transaminases, bilirrubina, gama glutamil transferase e fosfatase alcalina), sendo esses exames normais em todas. Nenhuma paciente tinha história pregressa de transtorno alimentar (anorexia nervosa, bulimia nervosa), abuso de álcool ou imobilização prolongada.

Das 33 pacientes previamente selecionadas, 9 foram excluídas de acordo com os seguintes critérios: uma teve o diagnóstico de hiperparatireoidismo primário, quatro pacientes tinham FSH > $40 \mathrm{U} / \mathrm{ml}$ compatível com menopausa espontânea, uma estava grávida, uma amamentando, uma apresentava deficiência de hormônio de crescimento confirmada ao ITT e uma negou-se a participar pois morava em outro estado.

Mediante anamnese e avaliação dos prontuários, foram extraídos dados como idade do diagnóstico da hiperprolactinemia para cálculo estimado do tempo de doença, características dos ciclos menstruais e os níveis de prolactina no ano anterior à densitometria óssea. O exame físico incluía a medida de peso e altura (com roupas leves e sem sapatos) para calcular o índice de massa corporal (IMC). 
Os exames de densitometria óssea foram realizados por um só operador, no mesmo aparelho Prodigy Advanced Lunar - GE, utilizando o mesmo software, versão 9.15, e analisados pela mesma médica com habilitação em Densitometria pela AMB/ Colégio Brasileiro de Radiologia/ Sociedade Brasileira de Densitometria Clínica e Certificação Técnica pela ISCD (International Society of Bone Densitometry). O coeficiente de variação (CV\%) é de 1,5\% para as regiões analisadas, determinado segundo os critérios das Sociedades Brasileira e Internacional de Densitometria, in vivo, com reposição entre os exames de 30 voluntários. Os sítios avaliados foram coluna lombar e fềmur proximal (colo, trocânter e fêmur total) e a densidade mineral óssea foi expressa em $\mathrm{g} / \mathrm{cm}^{2}$. De acordo com os atuais critérios da ISCD para análise de pacientes entre 20 e 50 anos, endossados pela Sociedade Brasileira de Densitometria Clínica, foram também considerados os Z-escores para todas as regiões analisadas. $\mathrm{O} \mathrm{Z}$-escore compara a massa óssea da paciente com a massa óssea de mulheres saudáveis pareadas por idade, etnia e índice de massa corpórea do banco de dados NHAMES III, e classifica como "densidade óssea abaixo do esperado" valores de Z-escores $\leq-2$ DP.

Utilizamos o teste $t$ de Student para amostras não pareadas ou o teste de Mann-Whitney para comparar subgrupos: macro versus microprolactinoma, prolactina média no ano anterior dentro da faixa normal versus elevada, e número de ciclos menstruais no último ano $\geq 8$ versus $<8$, neste último caso caracterizando oligo-amenorréia. As variáveis densitométricas foram relacionadas aos dados clínicos e bioquímicos pelo teste de correlação de Spearman. Utilizamos o intervalo de confiança de $95 \%$.

\section{RESULTADOS}

No momento do estudo, a idade das vinte e quatro pacientes foi de $36,6 \pm 10$ anos. A idade por ocasião do diagnóstico da hiperprolactinemia foi de 28,5 \pm 9,7 anos e o tempo estimado de doença de $8,4 \pm 6,2$ anos. De acordo com a cor da pele, dezenove se declararam brancas e cinco não brancas. Nove pacientes tinham o diagnóstico de microprolactinoma e quinze tinham macroprolactinoma. Oito pacientes tinham diagnostico de obesidade (IMC $>30 \mathrm{~kg} / \mathrm{m}^{2}$ ). Apenas uma era fumante.

$\mathrm{O}$ uso de agonista dopaminérgico era por vezes irregular, justificando a persistência de níveis elevados da prolactina sérica no ano anterior à densitometria na maioria delas: média e DP $=165,8 \pm 346 \mathrm{ng} / \mathrm{ml}$, mediana ( $1^{\circ}$ e $3^{\circ}$ quartis) $74,6 \mathrm{ng} / \mathrm{ml}(19,3$ e 103,3 $\mathrm{ng} / \mathrm{ml}$ ). Essa variabilidade no controle da hiperprolactinemia reflete-se no número de ciclos menstruais no ano anterior à densitometria óssea, com média 9,2 $\pm 3,9$ ciclos/ano, variando de zero (na única paciente que manifestou a doença como amenorréia primária) até menstruações regulares com 12 ciclos ao ano.

Nenhuma paciente referia fraturas prévias com pequeno impacto que pudessem ser consideradas por fragilidade, nem se queixava de dores ósseas. Entretanto, a densitometria mostrou redução da massa óssea em uma significativa parcela deste grupo. Assim, 20,83\% das pacientes apresentavam massa óssea na coluna lombar abaixo do esperado para mulheres da mesma idade, IMC e etnia. No fêmur proximal, nenhuma paciente apresentava Z-escore $\leq-2$ desvios-padrão.

A comparação dos dados clínicos e densitométricos entre os grupos de pacientes com macroprolactinoma e microprolactinoma não mostrou diferenças significativas, conforme demonstrado na tabela 1 .

Considerando os grupos com prolactina média no ano anterior à densitometria óssea dentro da faixa normal $(\mathrm{n}=8)$ versus prolactina elevada $(\mathrm{n}=16)$, vemos que somente as hiperprolactinêmicas apresentavam Z-escore na coluna $\leq-2 \mathrm{DP}$. A comparação desses grupos é mostrada na tabela 2. A correlação da

Tabela 1. Comparação entre as pacientes com macro e microprolacinoma.

\begin{tabular}{lccc}
\hline & Macroprolactinoma & Microprolactinoma & $\boldsymbol{p}$ valor \\
\hline Idade atual (anos) & $\mathrm{n}=15$ & $\mathrm{n}=9$ & \\
Idade diagnóstico (anos) & $35,7 \pm 10,4$ & $37,6 \pm 9,1$ & 0,658 \\
Tempo doença (anos) & $27,1 \pm 10,7$ & $30,9 \pm 7,9$ & 0,396 \\
PRL diagnóstico (ng/ml) & $9,6 \pm 6,7$ & $6,3 \pm 4,9$ & 0,244 \\
PRL último ano (ng/ml) & $134,5(114,3$ e 609,5$)$ & $101,3(98,6$ e 127$)$ & 0,146 \\
№ ciclos último ano & $79,9(21,8$ e 124,5$)$ & $53(18,7$ e 80,8) & 0,208 \\
IMC (kg/m²) & $8,1 \pm 4,5$ & $11,1 \pm 1,6$ & 0,086 \\
DMO L2-L4 (g/cm²) & $29 \pm 6,7$ & $25,9 \pm 6,4$ & 0,279 \\
DMO colo fêmur (g/cm²) & $1,114 \pm 0,214$ & $1,155 \pm 0,129$ & 0,604 \\
DMO fêmur total (g/cm²) & $1,003 \pm 0,121$ & $0,994 \pm 0,124$ & 0,861 \\
\hline
\end{tabular}

PRL: prolactina, IMC: índice de massa corporal, DMO: densidade mineral óssea.

Valores expressos como média \pm DP exceto a prolactina, expressa como mediana (intervalo interquartis). 
média de prolactina com a DMO nos sítios da coluna $(\mathrm{r}=-0,24, \mathrm{p}=0,26)$, colo femural $(\mathrm{r}=-0,17, \mathrm{p}=$ $0,45)$ e fêmur total $(\mathrm{r}=-0,19, \mathrm{p}=0,39)$ não foi estatisticamente significativa.

Dividindo as pacientes pelo número de ciclos menstruais no ano anterior à densitometria óssea, em $\geq$ $8(\mathrm{n}=19)$ versus $<8(\mathrm{n}=5)$, apenas uma do primeiro grupo tinha $\mathrm{Z}$-escore na coluna $\leq-2 \mathrm{DP}$, enquanto que três das cinco oligo-amenorrêicas apresentavam Z-escore $\leq-2$ DP nesta região. A comparação desses grupos é mostrada na tabela 3. Houve correlação positiva e significativa entre a DMO na coluna e o número de ciclos no ano anterior $(\mathrm{r}=0,515, p=0,017)$.

Observamos que, das cinco pacientes com Zescore na coluna $\leq-2 \mathrm{DP}$, duas eram obesas (IMC 37,3 e $40,3 \mathrm{~kg} / \mathrm{m}^{2}$ ). A densidade óssea no quadril foi positivamente influenciada pelo IMC, sendo as correlações significativas com a DMO do colo femural $(\mathrm{r}=0,563, p=0,006)$ e com a DMO no fềmur total $(\mathrm{r}=0,529, p=0,011)$.

\section{DISCUSSÃo}

Nossos resultados mostram redução da massa óssea em cerca de $20 \%$ das mulheres com prolactinoma, mesmo durante a menacme. Esse fato já havia sido descrito na literatura (3-5); entretanto, considerando a influência que a raça, a exposição solar, a alimentação e os fatores genéticos exercem sobre a massa óssea $(11,12)$, julgamos importante estender esta avaliação a pacientes com prolactinoma no Brasil.
Em 1993, Kayath e cols. foram os primeiros a avaliar a DMO em pacientes brasileiros com prolactinoma (13). No entanto, a análise contemplou ambos os sexos até 56 anos, incluindo mulheres em menopausa. Em 2005, Naliato e cols. descreveram elevada prevalência de doença óssea em pacientes brasileiros com prolactinoma, num estudo restrito a homens (14).

Nosso estudo concentrou-se em mulheres na menacme, evitando o efeito deletério da menopausa sobre a massa óssea. Outras características que tornaram nossa população mais homogênea foram os rigorosos critérios de exclusão, pois evitamos analisar pacientes com deficiência de hormônio do crescimento, hiperparatireoidismo primário ou secundário, doenças sistêmicas ou uso crônico de medicamentos que pudessem interferir na massa óssea. Embora não tenhamos dosado 25hidroxivitamina $\mathrm{D}$, todas as pacientes tinham cálcio e PTH séricos normais, tornando pouco provável uma deficiência de vitamina $\mathrm{D}$ clinicamente importante.

A densitometria óssea é o melhor método para quantificar a massa óssea e permite a classificação das pacientes como normal, osteopênica ou osteoporótica (15). No entanto, essa classificação da Organização Mundial de Saúde é válida para mulheres na pósmenopausa, não sendo adequado utilizá-la na fase prémenopausa. Durante a menacme, só podemos confirmar osteoporose quando existe fratura por fragilidade, o que não foi encontrado em nossas pacientes; por isso, evitamos classificá-las pelo T-escore.

Segundo recente consenso da International Society for Clinical Densitometry (16), em mulheres pré-menopausa e homens abaixo de 50 anos deve-se

Tabela 2. Comparação entre as pacientes que mantiveram prolactina sérica normal ou elevada no ano anterior ao estudo.

\begin{tabular}{|c|c|c|c|}
\hline & PRL normal & PRL elevada & $p$ valor \\
\hline & $n=8$ & $n=15$ & \\
\hline Idade atual (anos) & $35,1 \pm 8,7$ & $36,5 \pm 10,7$ & 0,756 \\
\hline Idade diagnóstico (anos) & $28,1 \pm 7,2$ & $27,6 \pm 10,6$ & 0,903 \\
\hline Tempo doença (anos) & $7 \pm 2,4$ & $9,9 \pm 7,4$ & 0,304 \\
\hline PRL diagnóstico $(\mathrm{ng} / \mathrm{ml})$ & $101,3(98,6$ e 123,3$)$ & $137(114,3$ e 467$)$ & 0,146 \\
\hline PRL último ano (ng/ml) & $18,7(17$ e 19,3$)$ & $89,4(76,3$ e 124,5$)$ & 0,0001 \\
\hline № ciclos último ano & $10,5 \pm 4$ & $8,5 \pm 3,7$ & 0,261 \\
\hline IMC $\left(\mathrm{kg} / \mathrm{m}^{2}\right)$ & $24,1 \pm 3$ & $29,4 \pm 7,3$ & 0,064 \\
\hline DMO L2-L4 (g/cm²) & $1,148 \pm 0,120$ & $1,118 \pm 0,218$ & 0,729 \\
\hline DMO colo fem $\left(\mathrm{g} / \mathrm{cm}^{2}\right)$ & $1,028 \pm 0,065$ & $0,984 \pm 0,141$ & 0,449 \\
\hline DMO fêmur total $\left(\mathrm{g} / \mathrm{cm}^{2}\right)$ & $1,093 \pm 0,125$ & $1,006 \pm 0,178$ & 0,270 \\
\hline
\end{tabular}

PRL: prolactina, IMC: índice de massa corporal, DMO: densidade mineral óssea.

Valores expressos como média \pm DP exceto a prolactina, expressa como mediana (intervalo interquartis).

Obs: não foi possível obter informações sobre a média de prolactina no último ano em 1 paciente, por isso foi excluída da análise. 
Tabela 3. Comparação entre as pacientes com oito ou mais ciclos menstruais no ano anterior ao estudo e as que permaneceram em oligo-amenorréia.

\begin{tabular}{|c|c|c|c|}
\hline & $\geq 8$ ciclos/ano & $<8$ ciclos/ano & $p$ valor \\
\hline & $n=16$ & $n=5$ & \\
\hline Idade atual (anos) & $36,8 \pm 9,5$ & $40 \pm 8,3$ & 0,501 \\
\hline Idade diagnóstico (anos) & $28,4 \pm 8,2$ & $29,4 \pm 11,2$ & 0,827 \\
\hline Tempo doença (anos) & $8,1 \pm 4,3$ & $10,6 \pm 10,4$ & 0,464 \\
\hline PRL diagnóstico (ng/ml) & $109,5(93,8$ e 169,3$)$ & $130(122$ e 3000$)$ & 0,154 \\
\hline PRL último ano (ng/ml) & $53(18,7$ e 92,3$)$ & $88,8(79,9$ e 105,5$)$ & 0,148 \\
\hline № ciclos último ano & $11,1 \pm 1,5$ & $3,2 \pm 3,1$ & 0,0001 \\
\hline $\mathrm{IMC}\left(\mathrm{kg} / \mathrm{m}^{2}\right)$ & $27,3 \pm 6,3$ & $27,7 \pm 7,2$ & 0,883 \\
\hline DMO L2-L4 $\left(\mathrm{g} / \mathrm{cm}^{2}\right)$ & $1,163 \pm 0,136$ & $0,988 \pm 0,177$ & 0,030 \\
\hline DMO colo fem $\left(\mathrm{g} / \mathrm{cm}^{2}\right)$ & $-0,3(-1$ e 0,3$)$ & $-2(-2,9$ e $-1,4)$ & 0,031 \\
\hline DMO fêmur total $\left(\mathrm{g} / \mathrm{cm}^{2}\right)$ & $1,026 \pm 0,108$ & $0,948 \pm 0,144$ & 0,209 \\
\hline
\end{tabular}

PRL: prolactina, IMC: índice de massa corporal, DMO: densidade mineral óssea.

Valores expressos como média \pm DP exceto a prolactina, expressa como mediana (intervalo interquartis).

Obs: não foi possível obter informações sobre o número de ciclos em três pacientes, por isso foram excluídas da análise.

preferir o termo "baixa massa óssea para a idade" quando o Z-escore é $\leq-2 \mathrm{DP}$, ou seja, a densidade óssea do paciente está $\leq-2$ DP abaixo do esperado para população do mesmo sexo, idade, etnia e IMC. Na região da coluna lombar, $20,83 \%$ das pacientes preenchiam esse critério, mas nenhuma paciente mostrou baixa massa óssea no quadril.

Nossos dados estão de acordo com outros autores, que também referem maior prejuízo da densidade óssea vertebral. Cann, em 1984, descreveu uma perda óssea na coluna lombar que variou de 20 a $30 \%$ em um grupo de 38 mulheres com amenorréia, 9 delas secundária à hiperprolactinemia. No mesmo ano, Koppelman e cols. avaliaram a DMO em coluna lombar de 13 mulheres com hiperprolactinemia comparando com grupo controle, e encontraram conteúdo mineral ósseo $10 \%$ menor nas pacientes com hiperprolactinemia (17). Posteriormente, Schlechte e cols. (3) estudaram 26 pacientes com prolactinoma e compararam a magnitude de perda óssea em sítios distintos; na coluna lombar, a massa óssea encontravase $25 \%$ menor nas mulheres com hiperprolactinemia em comparação ao grupo normal, enquanto no antebraço a massa óssea era semelhante entre os grupos.

Somente Kayath e cols. (13) descreveram perda óssea semelhante nas regiões da coluna lombar (13,4\% dos pacientes), colo femural $(15,5 \%)$ e trocânter $(11,1 \%)$ em 35 pacientes brasileiros de ambos os sexos com prolactinoma. No entanto, as características de sua amostra foram diferentes das nossas, pois incluiu ambos os sexos e havia pacientes em menopausa.
O IMC possui reconhecida influência na massa óssea, seja pela carga mecânica, principalmente em membros inferiores, seja pela maior síntese no tecido adiposo de estrona a partir de androstenediona e de estradiol a partir de testosterona (19). Em nossas pacientes, essa influência ficou demonstrada pela correlação entre IMC e DMO no colo femural e no fềmur total.

A DMO entre as pacientes com micro e macroprolactinoma foi semelhante. Alguns dados poderiam explicar o fato: 1) o critério rígido de inclusão segundo o qual todas as pacientes, mesmo as com microprolactino$\mathrm{ma}$, precisariam ter prolactina basal maior que $100 \mathrm{ng} / \mathrm{dl}$; assim, a média e a mediana da prolactina ao diagnóstico não diferiu entre micro e macroprolactinomas; 2) ambos os grupos encontravam-se em tratamento na ocasião da medida da densidade óssea, não havendo também diferença entre os níveis médios da prolactina no ano anterior ao exame; 3) o IMC era semelhante entre os grupos.

A comparação das pacientes que mantiveram prolactina normal e as que permaneceram hiperprolactinêmicas no ano anterior à densitometria não mostrou diferença nos parâmetros clínicos ou densitométricos. Talvez um ano tenha sido insuficiente para a avaliação, uma vez que o tempo de tratamento era mais longo. Entretanto, chamou a atenção que o número de ciclos menstruais fosse semelhante entre os grupos "bem" e "mal" controlados; isto é, a regularização dos ciclos menstruais parecia independer da média da prolactina no mesmo período de avaliação.

Sabe-se que o tratamento clínico com agonistas dopaminérgicos nem sempre permite pleno controle 
da hiperprolactinemia e restauração da função gonadal (20). Esse desequilíbrio hormonal pode comprometer a aquisição do pico de massa óssea ou antecipar para o menacme a perda óssea comum à fase pós-menopausa.

Em 1988, Klibanski e cols. (4) relacionaram a perda óssea encontrada nas mulheres hiperprolactinêmicas aos baixos níveis de estradiol e SDHEA, sugerindo que a insuficiência gonadal seria um fator primordial na perda óssea dessas pacientes. Em nosso grupo, utilizamos o número de ciclos menstruais anuais como parâmetro de função gonadal preservada. Classificamos como oligo-amenorrêicas as pacientes cujo número de ciclos era menor que 8 (intervalo entre as menstruações maior que 42 dias) e como ciclos regulares aquelas que apresentavam 8 ou mais ciclos no ano de análise (21). Constatamos que a densidade óssea e o Z-escore da coluna eram mais baixos no grupo com oligo-amenorréia. Esse padrão é semelhante ao encontrado na menopausa, com perda preponderante de osso trabecular.

Nossos resultados assemelham-se aos de Klibanski e cols., quando avaliaram a massa óssea no terço distal do rádio em 14 mulheres com hiperprolactinemia, 19 mulheres na pós-menopausa e 16 controles. Seus resultados mostraram perda óssea significativa no grupo com hiperprolactinemia, estando esses valores correlacionados aos níveis de estradiol, sem correlação com níveis séricos de prolactina (22). O efeito deletério da hiperprolactinemia mediado pelo hipoestrogenismo foi também demonstrado por Cicarelli e cols. (5) em um grupo de 22 pacientes com hiperprolactinemia: o conteúdo mineral ósseo na coluna lombar estava preservado naquelas que, apesar de hiperprolactinêmicas, mantinham ciclos menstruais regulares.

Concluímos que a redução na densidade mineral óssea é um achado freqüente em mulheres no menacme com prolactinoma, sendo a perda mais expressiva na coluna lombar. O IMC mostrou-se um fator protetor da massa óssea no fêmur proximal. Independentemente dos valores da prolactina no ano anterior à análise densitométrica, o maior determinante da densidade mineral óssea na coluna lombar foi o número de ciclos menstruais no mesmo período.

\section{REFERÊNCIAS}

1. National Institutes of Health Osteoporosis Prevention, Diagnosis and Therapy. National Institutes of Health Consensus Statement 2000;(17):1-45.

2. Miller KK, Klibanski A. Amenorrheic bone loss. J Clin Endocrinol Metab 1999;84:1775-83.

3. Schlechte J. Forearm and vertebral bone mineral in treated and untreated hyperprolactinemic amenorrhea. J Clin Endocrinol Metab 1987;64:1021-6.
4. Klibanski A, Biller BMK, Rosenthal DI, Schoenfeld A, Saxe V. Effects of prolactin and estrogen deficiency in amenorrheic bone loss. J Clin Endocrinol Metab 1988;67:124-30.

5. Ciccarelli E, Savino L, Carlevatto V, Bertagna A, Isaia GC, Camanni F. Vertebral bone density in non-amenorrheic hyperprolactinaemic women. Clin Endocrinol 1988;28:1-6.

6. Villar L, Gadelha MR, Une K. Avaliação diagnóstica da hiperprolactinemia. In: Vilar L, Kater CE (ed). Endocrinologia Clínica. $3^{\mathrm{a}}$ ed. Rio de Janeiro: Guanabara-Koogan, 2005. pp. 29-37.

7. Biller BMK, Baum HBA, Rosenthal DI, Saxe VC, Charpie PM, Klibanski A. Progressive trabecular osteopenia in women with hyperprolactinemic amenorrhea. J Clin Endocrinol Metab 1992;75:692-7.

8. Coss D, Yang L, Kuo CB, Xu X, Luben RA, Walker AM. Effects of prolactin on osteoblast alkaline phosphatase and bone formation in the developing rat. Am J Physiol Endocrinol Metab 2000;279:E1216-25.

9. Gagel RF. Multiple endocrine neoplasia. In: Williams RH, Wilson JD (ed). Williams Textbook of Endocrinology. 9th ed. Philadelphia: W.B. Saunders Company, 1998. pp. 1628-33.

10. Lanou AJ. Calcium and dairy products, and bone health in children and young adults: a re-evaluation of the evidence. Pediatrics 2005;115:736-43.

11. Ettinger B, Sidney S, Cummings SR, Libanati C, Bikle DD, Tekawa IS, et al. Racial differences in bone density between young adult black and white subjects persist after adjustment for anthropometric, lifestyle, and biochemical differences. J Clin Endocrinol Metab 1997;82:429-34.

12. Specker BL. Evidence for an Interaction between calcium intake and physical activity on changes in bone mineral density. J Bone Miner Res 1996;11:1539-44.

13. Kayath MJ, Lengyel AMJ, Vieira JGH. Prevalence and magnitude of osteopenia in patients with prolactinoma. Braz $\mathbf{J}$ Med Biol Res 1993;26:933-41.

14. Naliato EC, Farias ML, Braucks GR, Costa FS, Zylberberg D, Violante $\mathrm{AH}$. Prevalence of osteopenia in men with prolactinoma. J Endocrinol Invest 2005;28:12-7.

15. Lewiecki EM, Watts NB, McClung MR, Petak SM, Bachrach LK, Shepherd JA, et al; International Society for Clinical Densitometry. Official positions of the International Society for Clinical Densitometry. J Clin Endocrinol Metab 2004;89:3651-5.

16. Binkley N, Bilezikian JP, Kendler DL, Leib ES, Lewiecki EM, Petak SM. Official positions of the International Society for Clinical Densitometry and executive summary of the 2005 position development conference. J Clin Densitom 2006;9:4-14.

17. Cann EC, Martin MC, Jaffe RB. Decreased spinal mineral content in amenorrheic women. JAMA 1984;251(5):698-703.

18. Koppelman MCS, Kurtz DW, Morrish KA, Bou E, Susser JK, Shapiro JR, et al. Vertebral body bone mineral content in hyperprolactinemic women. J Clin Endocrinol Metab 1984; $59: 1050-3$.

19. Kaufman JM. Role of sex steroids in the regulation of bone metabolism in the adult skeleton. Ann Endocrinol 2006;67(2):119-22.

20. Gillam MP, Molitch ME, Lombardi G, Colao A. Advances in the treatment of prolactinomas. Endocr Rev 2006;27(5):485-534.

21. Hillard PJA, Deitch HR. Menstrual disorders in the collegeage female. Pediatr Clin North Am 2005;52:179-97.

22. Klibanski A, Neer RM, Beitins IZ, Ridgway EC, Zervas NT, McArthur JW. Decreased bone density in hyperprolactinemic women. N Engl J Med 1980;303:1511-4.

23. Biller BMK, Baun HBA, Rosenthal DI. Progressive trabecular osteopenia in women with hyperprolactinemic amenorrhea. J Clin Endocrinol Metab 1992;75:692-7.

\section{Endereço para correspondência:}

Isabela Bussade de Freitas Lopes

Rua General Tasso Fragoso 54, apto. 302

Rio de Janeiro, RJ

Fax: (21) 2527-1333

E-mail: ibussade@terra.com.br 